\title{
Psychosomatic symptoms during South East Asian Haze Crisis are related to changes in cerebral hemodynamics
}

Benjamin YQ Tan ${ }^{1}$, Nicholas JH Ngiam¹, M Sharma ${ }^{1}$, Leonard LL Yeo ${ }^{1}$, PA Seow ${ }^{1}$, CS Hong1, Elaine YH Chee ${ }^{1}$, JT Chen ${ }^{1}$, Hideyoshi Du Zhengdao ${ }^{1}$, Lily Wong ${ }^{1}$, HL Teoh ${ }^{1}$, Roger CM Ho ${ }^{2,3}$, Vijay K Sharma1,3

${ }^{1}$ Division of Neurology, Department of Medicine, National University Health System, Singapore 2Department of Psychological Medicine, National University Health System, Singapore

${ }^{3}$ Yong Loo Lin School of Medicine, National University of Singapore

\section{Background}

- Forest fires in South Asia periodically led to widespread haze for a few months per year in neighbouring countries.

- The magnitude of health effects of short-term haze exposure remains to be studied, but acute psychosomatic symptoms have been reported in previous studies during acute haze crises.

- We investigated the effect of haze exposure on cerebral haemodynamics and psychosomatic symptoms.

\section{Subjects \& Methods}

- We enrolled 74 healthy volunteers for this study. Baseline transcranial doppler (TCD) was performed by a trained sonographer in an indoor, air-conditioned environment.

- Each subject then had a second TCD performed by a second sonographer after thirty minutes outdoors.

- The corresponding prevailing level of pollutant standards index (PSI) was measured.

- Any new symptoms experienced by the participants were recorded by means of a standardized questionnaire.

- Appropriate statistical analyses were employed to compare cerebral hemodynamics between the symptomatic and asymptomatic groups of participants.

\section{Results}

-Our subjects had a median age of 30 (IQR 26-34) years, of which $60.8 \%$ were female.

-35 subjects developed psychosomatic symptoms.

-There was a significantly reduced pulsatility index (PI) in both the left and right middle cerebral arteries (MCA) for the symptomatic group compared to the asymptomatic group (left MCA: $0.85 \pm 0.10$ vs $0.93 \pm 0.07 p<0.001$; right MCA: $0.84 \pm 0.13$ vs $0.94 \pm 0.08, p<0.001$ ).

-In the symptomatic group, mean pulsatility index (PI) decreased from $0.90 \pm 0.12$ to $0.84 \pm 0.10(p<0.008)$ after haze exposure.

-There was a moderate negative correlation between $\mathrm{PI}$ and number of psychosomatic symptoms experienced $(r=0.519, p<0.001)$.

Table 1. Cerebral hemodynamic parameters obtained after haze exposure in asymptomatic and symptomatic participants $(n=74)$.

\begin{tabular}{|c|c|c|c|c|}
\hline & \multicolumn{4}{|c|}{ Univariate analysis } \\
\hline & $\begin{array}{c}\text { Asymptomatic } \\
(\mathrm{n}=39)\end{array}$ & $\begin{array}{c}\text { Symptomatic } \\
(\mathrm{n}=35)\end{array}$ & $\begin{array}{c}\text { Mean } \\
\text { difference }(95 \% \\
\text { CI) }\end{array}$ & p-value \\
\hline \multicolumn{5}{|c|}{ Left middle cerebral artery } \\
\hline $\begin{array}{l}\text { Mean flow velocity } \\
\text { (mean, SD) }\end{array}$ & $60.0(9.9)$ & $66.5(13.6)$ & $\begin{array}{c}-6.5(-12.1-- \\
1.0) \\
\end{array}$ & 0.022 \\
\hline $\begin{array}{l}\text { Peak systolic velocity } \\
\text { (mean, SD) }\end{array}$ & $97.1(16.3)$ & $104.2(20.4)$ & $-7.1(-15.7-1.5)$ & 0.106 \\
\hline $\begin{array}{l}\text { End diastolic velocity } \\
\text { (mean, SD) }\end{array}$ & $41.4(6.9)$ & $48.2(10.9)$ & $\begin{array}{c}-6.8(-11.1-- \\
2.5) \\
\end{array}$ & 0.003 \\
\hline Pulsatility index & $0.93(0.07)$ & $0.85(0.10)$ & $\begin{array}{c}0.08(0.04- \\
0.12)\end{array}$ & $<0.001$ \\
\hline Breath holding index & $1.6(0.6)$ & $1.7(0.5)$ & $-0.1(-0.3-0.2)$ & 0.471 \\
\hline \multicolumn{5}{|c|}{ Right middle cerebral artery } \\
\hline $\begin{array}{l}\text { Mean flow velocity } \\
\text { (mean, SD) }\end{array}$ & $60.7(11.4)$ & $63.9(12.3)$ & $-3.3(-8.8-2.2)$ & 0.241 \\
\hline $\begin{array}{l}\text { Peak systolic velocity } \\
\text { (mean, SD) }\end{array}$ & $98.6(17.3)$ & $99.3(18.9)$ & $-0.8(-9.2-7.7)$ & 0.860 \\
\hline $\begin{array}{l}\text { End diastolic velocity } \\
\text { (mean, SD) }\end{array}$ & $41.8(8.6)$ & $46.1(9.6)$ & $-4.3(-8.5--0.0)$ & 0.048 \\
\hline Pulsatility index & $0.94(0.08)$ & $0.84(0.13)$ & $\begin{array}{c}0.10(0.05- \\
0.15) \\
\end{array}$ & $<0.001$ \\
\hline Breath holding index & $1.5(0.5)$ & $1.7(0.5)$ & $-0.2(-0.4-0.1)$ & 0.140 \\
\hline
\end{tabular}

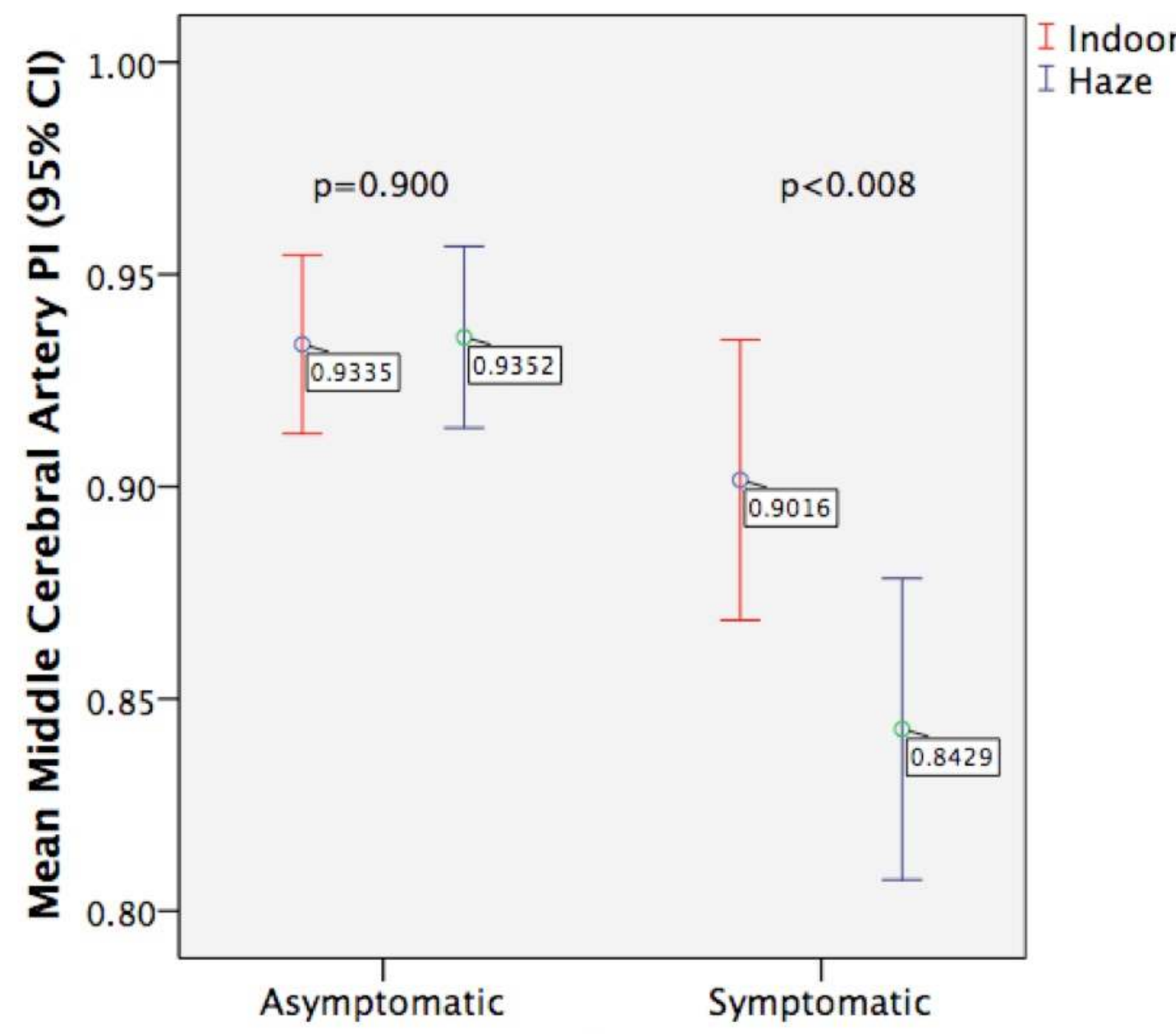

\section{Presence of Symptoms}

Figure 1: Paired sample T-tests demonstrating a significant difference $(p<0.008)$ between mean middle cerebral artery pulsatility index (PI) before and after haze exposure in the symptomatic group; however this difference is not seen in the asymptomatic group.

\section{Conclusion:}

Significant differences in cerebral haemodynamics as demonstrated by TCD exist after short-term haze exposure. The changes in PI highlight the possible cerebral vasodilatory effect of haze particles, which may play a role in causing psychosomatic symptoms. 